\title{
Expression of epithelial-mesenchymal transition markers in locally recurrent hepatocellular carcinoma after radiofrequency ablation
}

\author{
HIDEHIRO TAJIMA $^{1}$, TETSUO OHTA ${ }^{1}$, YASUHIRO SHOJI ${ }^{1}$, TOSHIFUMI WATANABE ${ }^{1}$, ISAMU MAKINO ${ }^{1}$, \\ HIRONORI HAYASHI ${ }^{1}$, HISATOSHI NAKAGAWARA ${ }^{1}$, ICHIRO ONISHI ${ }^{1}$, HIROYUKI TAKAMURA ${ }^{1}$, \\ ITASU NINOMIYA ${ }^{1}$, HIROHISA KITAGAWA ${ }^{1}$, SACHIO FUSHIDA ${ }^{1}$, TAKASHI TANI ${ }^{1}$, TAKASHI FUJIMURA ${ }^{1}$, \\ MASATO KAYAHARA ${ }^{1}$, KUNIAKI ARAI ${ }^{2}$, TATSUYA YAMASHITA ${ }^{2}$, SHUICHI KANEKO $^{2}$ and YOH ZEN ${ }^{3}$
}

\author{
Departments of ${ }^{1}$ Gastroenterologic Surgery, and ${ }^{2}$ Gastroenterology, Division of Cancer Medicine, \\ Graduate School of Medical Science, Kanazawa University, Kanazawa, Japan; \\ ${ }^{3}$ Institute of Liver Studies, King's College Hospital, London, UK
}

Received December 4, 2009; Accepted February 1, 2010

DOI: 10.3892/etm_00000054

\begin{abstract}
Radiofrequency ablation (RFA) is reported to be a minimally invasive and effective procedure for treating hepatocellular carcinoma (HCC). However, some cases showing rapid and aggressive recurrence of HCC after RFA have recently been reported. We reviewed 15 resected-HCC patients who developed local recurrence after RFA and in whom tumor dedifferentiation could have been induced by RFA. Additionally, increasing evidence has shown that epithelial-mesenchymal transition (EMT) mediates tumor progression. As EMT is a key event in the tumor invasion process, we compared the immunohistochemical expression of mesenchymal markers, i.e., loss of E-cadherin and gain of $\mathrm{N}$-cadherin and vimentin, in 15 patients with local recurrence of HCC after RFA to those in 26 (control) HCCs without local therapy. The percentage of E-cadherin expression loss was significantly higher in the post-RFA cases $(60 \%)$ than in controls $(19.2 \%)(\mathrm{P}=0.02)$. Although no significant differences were observed in $\mathrm{N}$-cadherin and vimentin expression, vimentin was found only in cases with recurrence after RFA. These findings suggest that heat shock due to RFA may induce EMT of HCC, and that EMT is closely related to aggressive recurrence and dedifferentiation of HCC after RFA.
\end{abstract}

\section{Introduction}

Recently, local therapies for hepatocellular carcinoma (HCC), including percutaneous radiofrequency ablation (RFA),

Correspondence to: Dr Hidehiro Tajima, Department of Gastroenterologic Surgery, Division of Cancer Medicine, Graduate School of Medical Science, Kanazawa University, 13-1 Takaramachi, Kanazawa 920-8641, Japan

E-mail: hidetajima@surg2.m.kanazawa-u.ac.jp

Key words: hepatocellular carcinoma, epithelial mesenchymal transition, local recurrence, radiofrequency ablation have come to be widely employed. RFA has gained great popularity as a treatment technique for small HCC because of its feasibility, effectiveness, repeatability and safety (1-6). In most HCC patients, treatment options are limited by liver dysfunction due to underlying chronic inflammation and cirrhosis. Although complete surgical resection of HCC offers the best chance of long-term survival, cirrhosis may limit the extent of parenchymal resection that can be tolerated, and also increases the risk of postoperative liver failure and death (7). There is no significant difference in overall or diseasefree survival between surgical resection and RFA when HCC patients are in Child-Pugh class B (8). However, some cases experiencing rapid and aggressive $\mathrm{HCC}$ recurrence after RFA have recently been reported (9-11). In a previous study, we reviewed 15 resected-HCC patients who developed local recurrence after RFA and discussed the possibility of tumor dedifferentiation being induced by RFA (12).

Increasing evidence indicates that epithelial-mesenchymal transition (EMT) mediates tumor progression. EMT is a key event in the tumor invasion process, whereby epithelial cell layers lose polarity and cell-cell contacts and undergo a dramatic remodeling of the cytoskeleton (13). The hallmark of EMT is loss of E-cadherin (13) while $\mathrm{N}$-cadherin is expressed (the so-called cadherin switch) and vimentin expression in epithelial cells, which is accompanied by loss of tight cell-cell adhesion and acquisition of a fibroblastic morphology (14).

This study aimed to examine the expression of mesenchymal markers, E-cadherin, $\mathrm{N}$-cadherin and vimentin, in patients with local recurrence of HCC after RFA.

\section{Materials and methods}

Patients. Between January 2000 and December 2006, 174 HCC patients underwent surgery at the Department of Gastroenterologic Surgery of Kanazawa University Hospital (Kanazawa, Japan). Among them, 15 patients, 12 men and 3 women with an average age of 62.5 years (range 44-79 years), developed local HCC recurrence after RFA. Twenty-six 
Table I. Expression of EMT markers.

\begin{tabular}{lccc}
\hline EMT markers & Local recurrence after RFA $(\mathrm{n}=15)$ & Without local therapy $(\mathrm{n}=26)$ & P-value \\
\hline Loss of E-cadherin & $9(60.0 \%)$ & $5(19.2 \%)$ & 0.02 \\
N-cadherin & $14(93.3 \%)$ & $20(76.9 \%)$ & NS \\
Vimentin & $3(20.0 \%)$ & $0(0.0 \%)$ & 0.08 \\
\hline
\end{tabular}

EMT, epithelial-mesenchymal transition; RFA, radiofrequency ablation; NS, not significant.

cases who underwent HCC resection without local therapy and were diagnosed with classical HCC by preoperative radiological studies were selected as controls. All the patients gave their informed consent for their samples to be used for research purposes.

Pathological specimens. Formalin-fixed and paraffinembedded specimens were retrieved from the surgical pathology files of the Pathology Department of Kanazawa University Hospital.

Immunohistochemical examination. For immunohistochemical staining, the Dako Envision system, which uses dextran polymers conjugated with horseradish peroxidase (Dako, Carpinteria, CA, USA) was employed to avoid any endogenous biotin contamination. Tissues were fixed with $10 \%$ formaldehyde in phosphate-buffered saline, embedded in paraffin and cut into $5-\mu \mathrm{m}$ tissue sections. The sections were deparaffinized in xylene and rehydrated in a graded ethanol series. Endogenous peroxidase was blocked by immersing the sections in $3 \% \mathrm{H}_{2} \mathrm{O}_{2}$ in $100 \%$ methanol for $20 \mathrm{~min}$ at room temperature. Antigen retrieval was achieved by microwaving sections at $95^{\circ} \mathrm{C}$ for $10 \mathrm{~min}$ in $0.001 \mathrm{M}$ citrate buffer ( $\mathrm{pH}$ 6.7). After blocking the endogenous peroxidase, the sections were incubated with Protein Block Serum-Free (Dako) at room temperature for 10 min to block non-specific staining, and the sections were then incubated for $2 \mathrm{~h}$ at room temperature with 1:50 diluted mouse monoclonal antibodies against E-cadherin (Takara, Ohtsu, Japan), N-cadherin (Takara) and vimentin (Santa Cruz Biotechnology, Santa Cruz, CA, USA). Peroxidase activity was detected with enzyme substrate 3-amino-9-ethyl carbazole. For negative controls, the sections were incubated with Tris-buffered saline without the primary antibodies. Samples in which at least $10 \%$ of tumor cells were slightly counterstained with Mayer's hematoxylin were defined as positive.

Statistical analysis. Categorical variables were compared using the Chi-square test. For statistical analysis, P-values were calculated using a two-tailed test, and $\mathrm{P}<0.05$ was considered to indicate statistical significance.

\section{Results}

Fifteen surgically resected specimens of locally recurrent HCC after RFA were immunohistochemically examined for E-cadherin, $\mathrm{N}$-cadherin and vimentin expression and compared to those of $26 \mathrm{HCC}$ control specimens without local therapy (Table I).
E-cadherin. In 9 of the 15 (60\%) locally recurrent post-RFA HCCs, loss of E-cadherin expression was noted. By contrast, decreased E-cadherin expression was observed in only 5 of the $26(19.2 \%)$ controls. The percentage of cases negative for E-cadherin expression was significantly higher in the recurrent post-RFA HCC than in the control cases $(\mathrm{P}=0.02)$ (Fig. 1).

$N$-cadherin. Immunohistochemical analyses revealed membranous $\mathrm{N}$-cadherin expression in all non-tumoral liver tissues. $\mathrm{N}$-cadherin was expressed in hepatocytes and interlobular bile duct epithelia. Immunoreactivity was increased in 14 of the $15(93.3 \%)$ locally recurrent post-RFA HCCs. However, 20 of the 26 (76.9\%) control HCCs also showed N-cadherin immunoreactivity. No difference was observed in the intensity or the range of staining. In addition, there was no association between $\mathrm{E}$ - and $\mathrm{N}$-cadherin expression patterns, the so-called cadherin switch (Fig. 2).

Vimentin. Vimentin was found to be diffusely positive in the tumor cell cytoplasm in 3 of the 15 (20\%) post-RFA recurrent HCCs. In the control group, all 26 cases were negative for vimentin. The percentage of the cases positive for vimentin was not significantly higher in the locally recurrent post-RFA HCCs than in the controls $(\mathrm{P}=0.08)$. In non-cancerous areas, vascular endothelial cells and fibroblasts were positive for vimentin, while hepatocytes were negative (Fig. 3).

\section{Discussion}

RFA has become a very popular technique for the treatment of small HCC because of its feasibility, effectiveness, repeatability and safety (1-6). However, cases experiencing rapid and aggressive recurrence of HCC after RFA have recently been reported (9-11). Moreover, an autopsy case was reported in which well-differentiated HCC developed into HCC with sarcomatous change after RFA (15). In a previous study, we reviewed 15 resected-HCC patients who developed local recurrence after RFA. In patients with local HCC recurrence after RFA, the tumors showed more invasive growth, more vascular invasion and less differentiation than those in patients without RFA. We thus concluded that tumor dedifferentiation can be induced by the heat stress associated with RFA (12).

Recently, increasing evidence has shown that EMT, a process first identified in embryogenesis (16), mediates tumor progression, including local invasion, spread through the circulatory system and metastasis. Hallmarks of EMT include the loss of E-cadherin expression and the expression 
A

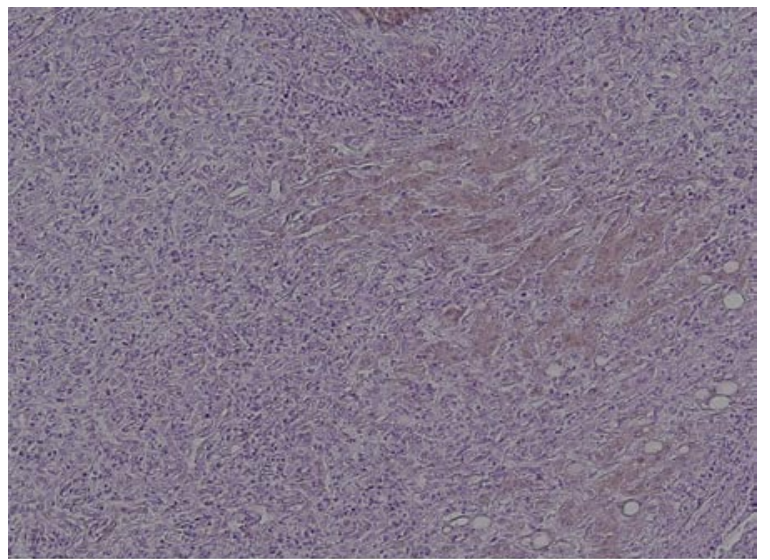

B

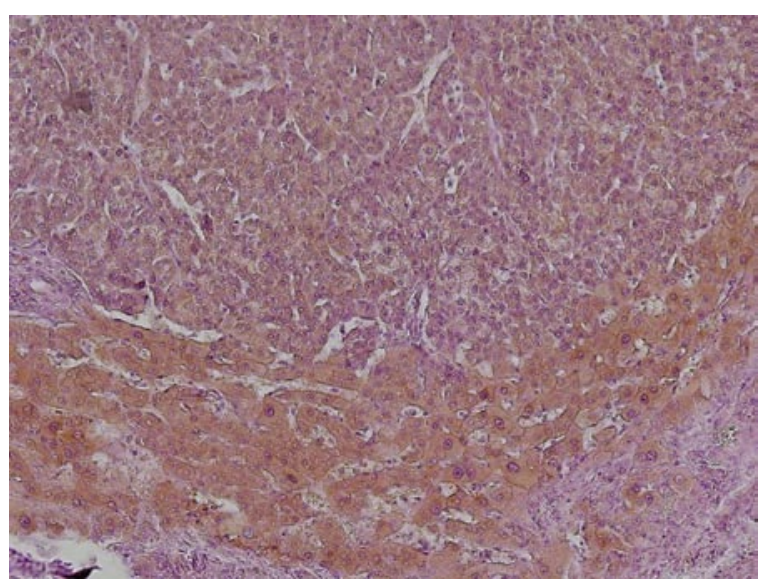

Figure 1. Immunohistochemistry of E-cadherin in hepatocellular carcinoma and adjacent non-tumor tissue. (A) Loss of E-cadherin expression in poorly differentiated hepatocellular carcinoma compared with expression in adjacent hepatocytes. (B) E-cadherin expression in moderately differentiated hepatocellular carcinoma and adjacent non-tumor tissues.

of mesenchymal markers, such as $\mathrm{N}$-cadherin and vimentin, in epithelial cells $(13,14)$.

E-cadherin, a transmembranous glycoprotein that mediates adherens junctions, is developmentally restricted to polarized epithelial cells (17). E-cadherin plays an essential role in maintaining the structural integrity and polarization of the epithelia. Its loss consequently allows destabilization of the structural integrity of the epithelium and causes cells to dissociate from their neighbors and lose polarity, as observed during gastrulation in the embryo and also during wound repair and metastasis of carcinoma cells (17). Decreased E-cadherin immunoreactivity correlates with a lack of differentiation, tumor aggressiveness and inhibited formation of nascent junctional complexes among HCC cells (18). On the other hand, E-cadherin has been recognized as an important tumor biomarker and has been identified as a bona fide tumor suppressor gene in diffuse gastric carcinomas (19).

$\mathrm{N}$-cadherin, found primarily in neural tissues and fibroblasts (20), is expressed in the most invasive and dedifferentiated breast cancer cell lines and in poorly differentiated areas of prostate cancers where E-cadherin expression is negative or aberrant. Exogenous N-cadherin in tumor cells enhances cellular motility, invasion and metastasis (21). Generally, cadherin subtype switching from E- to N-cadherin in tumors, not only activates a signaling program that

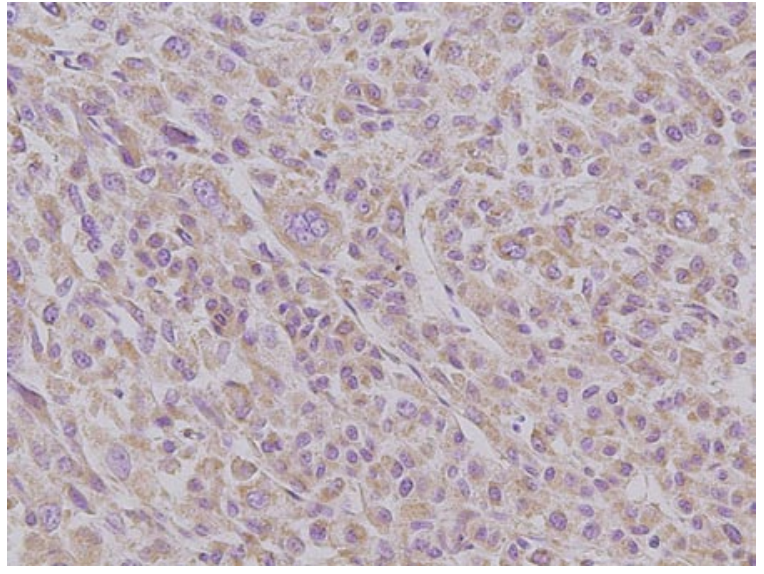

Figure 2. Immunohistochemistry of N-cadherin in hepatocellular carcinoma. Tumor cell membrane and cytoplasm expression of $\mathrm{N}$-cadherin.

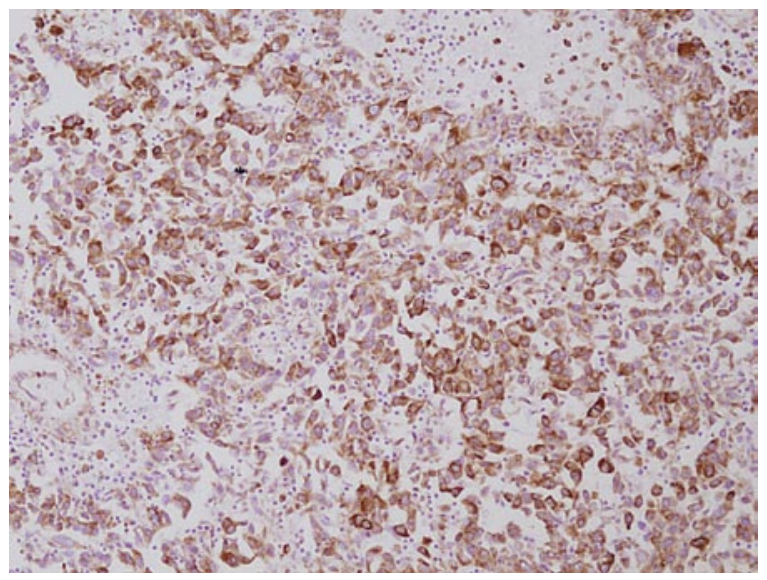

Figure 3. Immunohistochemistry of vimentin in hepatocellular carcinoma. Vimentin expression in poorly differentiated hepatocellular carcinoma with sarcomatous change.

promotes the invasive and survival capabilities of tumor cells, but also fosters cooperation between tumor cells and the surrounding microenvironment, a critical event in metastatic progression (22). However, an early study showed N-cadherin to be expressed in both normal liver and HCC tissues (23). Moreover, a recent study identified $\mathrm{N}$-cadherin as frequently being overexpressed in HCC, and loss of E-cadherin expression was not apparently a prerequisite for $\mathrm{N}$-cadherin expression (21). In the present study, we found no correlation between the expression patterns of E-cadherin and $\mathrm{N}$-cadherin in either the post-RFA or control cases with recurrence.

Vimentin, a cytoplasmic intermediate filament, is characteristic of mesenchymal cells and is not usually expressed in epithelial cells. The atypical expression of vimentin in epithelial cancer cells may be associated with local invasiveness and the potential for metastasis $(24,25)$. In the present study, although no statistically significant difference was observed, vimentin expression was found only in post-RFA recurrent HCCs $(\mathrm{P}=0.08)$.

Cancer cells suffer various forms of stress from anticancer drugs, irradiation, hypo-oxygenation, hypo-nutrition and heat treatment. Some recent studies suggest that these stresses 
induce EMT of cancer cells $(26,27)$, and that this EMT is related to the invasive potential of cancer cells (26). Moreover, heat stress reportedly induces the tyrosine phosphorylation and activation of a human carcinoma dedifferentiation modulator (28). Obara et al reported that even a single heat treatment may induce transformation of an $\mathrm{HCC}$ cell line and suggested that insufficient RFA treatment of HCC may induce further malignant transformation in vivo (29). Accordingly, the thermal effect of RFA may increase the risk of EMT and the transformation of residual viable tumor cells.

In conclusion, to the best of our knowledge, the present study is the first description of EMT induction by heat stress associated with RFA. Based on our findings, we suggest that EMT is closely related to aggressive recurrence and dedifferentiation of HCC after RFA. The impact of the complications reported herein may temper the optimistic outcomes reported in some initial series (30), suggesting that caution is needed when determining the indications for and applications of RFA, particularly in patients who are also appropriate surgical candidates. The risk of aggressive recurrence after RFA must be considered when assessing the treatment options for HCC. Further experimental research is needed before final conclusions can be drawn.

\section{References}

1. Rossi S, Garbagnati F, Lencinoni R, et al: Percutaneous radiofrequency thermal ablation of non-resectable hepatocellular carcinoma after occlusion of tumor blood supply. Radiology 217: 119-126, 2000.

2. Livraghi T, Goldberg SN, Lazzaroni S, et al: Small hepatocellular carcinoma: treatment with radio-frequency ablation versus ethanol injection. Radiology 210: 655-661, 1999.

3. Allgaier HP, Deibert P, Zuber I, et al: Percutaneous radiofrequency interstitial thermal ablation of small hepatocellular carcinoma. Lancet 353: 1676-1677, 1999.

4. Nicoli N, Casaril A, Marchiori L, et al: Intraoperative and percutaneous radiofrequency thermal ablation in the treatment of hepatocellular carcinoma. Chir Ital 52: 29-40, 2000.

5. Nicoli N, Casaril A, Marchiori L, et al: Treatment of recurrent hepatocellular carcinoma by radio-frequency thermal ablation. J Hepatobil Pancreat Surg 8: 417-421, 2001.

6. Solbaiati L, Livraghi T, Goldberg SN, et al: Percutaneous radiofrequency ablation of hepatic metastasis from colorectal cancer: long-term result in 117 patients. Radiology 221: 159-166, 2001.

7. Steven AC, Francesco I, Lee ME, Nicolas V and Paolo V: Radiofrequency ablation of hepatocellular cancer in 110 patients with cirrhosis. Ann Surg 232: 381-391, 2000.

8. Vivarelli M, Guglielmi A, Ruzzenente A, et al: Surgical resection versus percutaneous radiofrequency ablation in the treatment of hepatocellular carcinoma on cirrhotic liver. Ann Surg 204: 102-107, 2004.

9. Nicola N, Andrea C, Moh'd AH, et al: A case of rapid intrahepatic dissemination of hepatocellular cartinoma after radiofrequency thermal ablation. Am J Surg 188: 165-167, 2004.

10. Nazario P, Guido AMT, Maurizio R, et al: Aggressive recurrence after radiofrequency ablation of liver neoplasms. Hepatogastroenterology 50: 2179-2184, 2003.
11. Takada Y, Kurata M and Ohkohchi N: Rapid and aggressive recurrence accompanied by portal tumor thrombus after radiofrequency ablation for hepatocellular carcinoma. Int Clin Oncol 8: 332-335, 2003.

12. Tajima H, Ohta T, Okamoto K, et al: Radiofrequency ablation induces dedifferentiation of hepatocellular carcinoma. Oncol Lett 1: 91-94, 2010.

13. Yang J, Mani SA, Donaher JL, et al: Twist, a master regulator of morphogenesis, plays an essential role in tumor metastasis. Cell 117: 927-939, 2004.

14. Hay ED and Zuk A: Transformations between epithelium and mesenchyme: normal, pathological and experimentally induced. Am J Kidney Dis 26: 678-690, 1995.

15. Koda M, Maeda Y, Matsunaga Y, et al: Hepatocellular carcinoma with sarcomatous change arising after radiofrequency ablation for well-differentiated hepatocellular carcinoma. Hepatol Res 27: 163-167, 2003.

16. Soo K, O'Rourke MP, Khoo PL, et al: Twist function is required for the morphogenesis of the cephalic neural tube and the differentiation of the cranial neural crest cells in the mouse embryo. Dev Biol 247: 251-270, 2002.

17. Gumbiner BM: Cell adhesion: the molecular basis of tissue architecture and morphogenesis. Cell 84: 345-357, 1996.

18. Du GS, Lu JX, Ma CQ, et al: Expression of P-aPKC-I, E-cadherin and $\beta$-catenin related to invasion and metastasis in hepatocellular carcinoma. Ann Surg Oncol 16: 1578-1586, 2009.

19. Perl AK, Wilgenbus P, Dahl U, et al: A causal role for E-cadherin in the transition from adenoma to carcinoma. Nature 392: 190-193, 1998.

20. Hatta $\mathrm{K}$ and Takeichi M: Expression of N-cadherin adhesion molecules associated with early morphogenetic events in chick development. Nature 320: 447-449, 1986.

21. Seo DD, Lee HC, Kim HJ, et al: Neural cadherin overexpression is a predictive marker for early postoperative recurrence in hepatocellular carcinoma patients. J Gastroenterol Hepatol 23: 1112-1118, 2008.

22. Hazan RB, Qiao R, Keren R, Badano I and Suyama K: Cadherin switch in tumor progression (Review). Ann NY Acad Sci 1014: 155-163, 2004.

23. Ihara A, Koizumi H, Hashizume R and Uchikoshi T: Expression of epithelial cadherin and alpha- and beta-catenins in nontumoral livers and hepatocellular carcinomas. Hepatology 23: 1441-1447, 1996.

24. Kakizoe S, Kojiro $\mathbf{M}$ and Nakashima T: Hepatocellular carcinoma with sarcomatous change. Cancer 59: 310-316, 1987.

25. Park MY, Kim K, Park HS, et al: Expression of the serum response factor in hepatocellular carcinoma: Implications for epithelial-mesenchymal transition. Int J Oncol 31: 1309-1315, 2007.

26. Takamoto H, Shibata K, Kajiyama H, et al: Irradiation-induced epithelial-mesencymal transition (EMT) related to invasive potential in endometrial carcinoma cells. Gynecol Oncol 107: 500-504, 2007.

27. Yang AD, Fan F, Camp ER, et al: Chronic oxaliplatin resistance induces epithelial-mesenchymal transition in colorectal cancer cell lines. Clin Cancer Res 12: 4147-4153, 2006.

28. Yang SD, Lee SC and Chang HC: Heat stress induces tyrosine phosphorylation/activation of kinase FA/GSK-3 alpha (a human carcinoma dedifferentiation modulator) in A431 cells. J Cell Biochem 66: 16-26, 1997.

29. Obara K, Matsumoto N, Okamoto M, et al: Insuffcient radiofrequency ablation therapy may induce further malignant transformation of hepatocellular carcinoma. Hepatol Int 2: 116-123, 2008.

30. Llovet JM, Vilana R, Brú C, et al: Increased risk of tumor seeding after percutaneous radiofrequency ablation for single hepatocellular carcinoma. Hepatology 33: 1124-1129, 2001. 